\title{
Effects of aspect ratio and inclination angle on aerodynamic loads of a flat plate
}

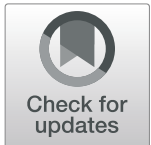

Mehrdad Shademan * (D) and Arash Naghib-Lahouti

\author{
* Correspondence: mehrdadkbg@ \\ gmail.com \\ Institute for Aerospace Studies, \\ University of Toronto, M3H5T6, \\ Toronto, Ontario, Canada
}

\begin{abstract}
Large Eddy Simulations are carried out to analyze flow past flat plate in different configurations and inclinations. A thin flat plate is considered at three inclination angles $\left(\alpha=30^{\circ}, 60^{\circ}\right.$ and $\left.90^{\circ}\right)$ and three aspect ratios ( $A R=0.5,2$ and 5). The Reynolds number based on the free stream velocity and chord length of the plate at different inclination angles varies between 75,000 to 150,000 . An increase in the inclination angle while the aspect ratio (span to chord) is constant results in higher drag and lower lift on the plate. Increasing the aspect ratio at a constant inclination angle increases the mean aerodynamic loading except for the $a=30^{\circ}$ and $A R=0.5$ case where the mean forces are larger than the other aspect ratios for this specific inclination angle. The small aspect ratio suppresses and blocks the separation of the flow from the top and bottom edges causing larger aerodynamic forces relative to $A R=2,5$. Visualization of the flow structures shows the tip vortices have a significant role in controlling the shedding vortices from the top and bottom edges. At $a=30^{\circ}$ and $A R=0.5$, the two tip vortcies control and suppress the flow separation from the top and bottom edges. A stable wake was found for this case with no fluctuation. As the aspect ratio increases, the influence of the tip vortices on flow separation from the top and bottom edges reduces. As a result, larger fluctuations were found for cases with higher aspect ratios.
\end{abstract}

Keywords: Flat plate, Aspect ratio, Inclination angle, CFD, OpenFOAM, Aerodynamic loads

\section{Introduction}

\subsection{Experimental studies}

The flow over inclined flat plates has been the subject of many studies over the past century. This is partly due to the fundamental nature of the problem. In many ways it is considered to be the simplest possible bluff body, because it is associated with fixed separation points and minimized distance between the windward side of the body and the wake region. Moreover, from a practical standpoint, this problem is a simplified representation of many modern day wind engineering applications such as solar panels exposed to extreme winds. This has resulted in renewed interest in investigating this flow problem using state-of-the-art experimental and computational tools.

(c) The Author(s). 2020 Open Access This article is licensed under a Creative Commons Attribution 4.0 International License, which permits use, sharing, adaptation, distribution and reproduction in any medium or format, as long as you give appropriate credit to the original author(s) and the source, provide a link to the Creative Commons licence, and indicate if changes were made. The images or other third party material in this article are included in the article's Creative Commons licence, unless indicated otherwise in a credit line to the material. If material is not included in the article's Creative Commons licence and your intended use is not permitted by statutory regulation or exceeds the permitted use, you will need to obtain permission directly from the copyright holder. To view a copy of this licence, visit http://creativecommons.org/licenses/by/4.0/. 
Previous studies investigate the effects of different geometric parameters such as the aspect ratio (span to chord ratio), inclination angle, planform shape and blockage effects, as well as free stream velocity, on aerodynamic characteristics of inclined flat plates. Table 1 represents a summary of the previous experimental studies on flow over flat plate.

\subsubsection{Effect of inclination angle and aspect ratio}

Among the experimental studies carried out on flow around inclined flat plate, the experiments by Fage and Johansen [1] have gained significant recognition as a benchmark, due to the comprehensive range of parameters investigated therein. Their experiments dealt with an infinite span plate at different inclination angles in which the behaviors of lift, drag, pressure coefficients, and shedding frequency were investigated. The infinite span term used here refers to a very large span to chord ratio configuration where the two ends of the plate are attached to the tunnel side walls. They performed the experiments at 18 inclination angles ranging from $0.15^{\circ}$ to $90^{\circ}$ at a speed of $15 \mathrm{~m} / \mathrm{s}$. The corresponding Reynolds number based on the chord length of the flat plate at $90^{\circ}$ inclination angle was 150,000 . Their results quantify the variation of pressure on the windward and leeward sides of the flat plate with increasing angle of incidence, and indicate that the maximum pressure difference between the windward and leeward surfaces occurs when the plate is positioned normal to the flow (i.e., at a $90^{\circ}$ incidence angle).

In another set of experiments, they measured the effect of different inclination angles (ranging between $12^{\circ}$ to $90^{\circ}$ ) on the shedding frequency. They noticed an increasing trend for the shedding frequency when the inclination angle increased from $12^{\circ}$ to $14^{\circ}$. For the rest of the angles from 14 to 90 degrees, a decreasing trend was observed for the shedding frequency.

Table 1 A summary of previous experimental studies on flow over inclined flat plate

\begin{tabular}{lllll}
\hline Experiments & $\begin{array}{l}\text { Aspect Ratio } \\
\text { (AR) }\end{array}$ & $\begin{array}{l}\text { Thickness/ } \\
\text { Chord }\end{array}$ & $\begin{array}{l}\text { Reynolds (based on chord length and } \\
\text { inlet velocity) }\end{array}$ & $\begin{array}{l}\text { Inclination } \\
\text { angle }\end{array}$ \\
\hline Fage and Johansen [1] & 14.1 (infinite) & 0.03 & 150,000 & $\begin{array}{c}0.15^{\circ} \sim 90^{\circ} \\
12^{\circ} \sim 90^{\circ}\end{array}$ \\
Flaschbart [2] & $50 \sim 200$ & $0.015 \sim 0.05$ & $1000 \sim 6000$ & $90^{\circ}$ \\
$\begin{array}{l}\text { Schubauer and Dryden } \\
\text { [3] }\end{array}$ & 6 & - & $30,000 \sim 60,000$ & $90^{\circ}$ \\
Roshko [4] & 13.5 & 0.16 & $4000 \sim 10,000$ & $90^{\circ}$ \\
Arie and Rouse [5] & 12 & 0.083 & 75,000 & $90^{\circ}$ \\
$\begin{array}{l}\text { Fail, Lawford and Eyre } \\
\text { [6] }\end{array}$ & $1 \sim 20$ & - & 80,000 & $90^{\circ}$ \\
Abernathy [7] & $1.5 \sim 6$ & $0.06 \sim 0.19$ & $36,000 \sim 140,000$ & $40^{\circ} \sim 90^{\circ}$ \\
Castro [8] & 15 & 0.04 & $25,000 \sim 90,000$ & $90^{\circ}$ \\
Sarpkaya \& Kline [9] & 8 & 0.11 & 21,000 & $90^{\circ}$ \\
Kiya and Matsumura & 15 & 0.215 & 23,000 & $90^{\circ}$ \\
[10] & & & & $90^{\circ}$ \\
$\begin{array}{l}\text { Mazharoglu and } \\
\text { Hacisevki [1 1] }\end{array}$ & 14 & 0.2 & 33,000 & $90^{\circ}$ \\
Xu et al. [12] & 12.1 & 0.125 & 2240 & \\
\hline
\end{tabular}


To evaluate the influence of the free stream velocity on the shedding frequency, they performed measurements on a normal flat plate (90 degrees) at free stream velocities ranging from 3.2 to $18 \mathrm{~m} / \mathrm{sec}$. The results showed that increasing the free stream velocity significantly increases the shedding frequency. At the speed of 3.2 $\mathrm{m} / \mathrm{sec}$ the shedding frequency was $3.13 \mathrm{~Hz}$, however, by increasing the velocity to $18 \mathrm{~m} / \mathrm{s}$, the shedding frequency increased to $17.35 \mathrm{~Hz}$. The Strouhal number based on the shedding frequency, incoming velocity and chord length remained constant at about 0.14 .

Effect of different aspect ratios on the flow around inclined flat plate has been investigated by many researchers as listed in Table 1. One of the most comprehensive studies of this effect is the study by Fail et al. [6], who performed wind tunnel tests at a speed of $43 \mathrm{~m} / \mathrm{sec}$ at aspect ratios ranging from 1 to 20 . Based on their definition, the main feature of the flow around a normal flat plate is a closed bubble behind the plate (An example of the generated bubble behind the plate can be observed in Fig. 1). The bubble is defined as the closed part of the mean flow streamlines behind the plate [6].

Their results indicate that as the aspect ratio increases, the drag increases, the base pressure and the bubble length behind the plate decrease. They concluded that these changes are small up to an aspect ratio of 10 and thereafter a more rapid change was observed. They also studied the effect of the planform geometry of the inclined flat plate by investigating square, circular and equilateral-triangular planforms of the same aspect ratio, on vortex shedding frequency and drag, and showed that vortex shedding frequency and drag coefficient are functions of the wake width (as defined by the separation between the two shear layers) rather than the planform geometry of the wake generator.

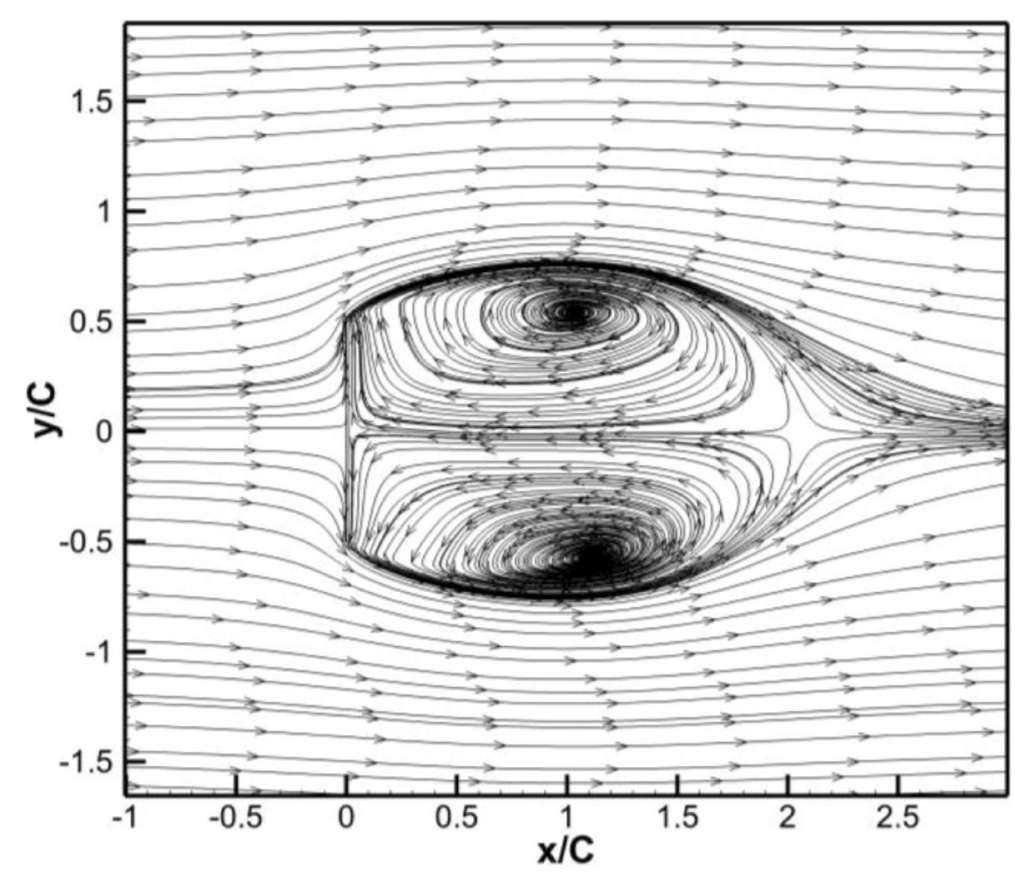

Fig. 1 Schematic of a bubble generated behind a normal flat plate (obtained from current simulation at $\operatorname{Re}=150,000$ ) 


\subsection{Numerical simulations}

With invention and increasing acceptance of numerical simulation as a capable tool for analysis of complex flows, researchers have used this tool to investigate various aspects of flow around flat plate. The growing capability of computers has strengthened the use of numerical methods. Using the new numerical algorithms by means of supercomputers, scientists are able to simulate highly complex flows and capture the evolution of different flow parameters. Examples of these include Large Eddy and Direct Numerical Simulations (LES, DNS) which present time resolved information of the flow field. Additionally, numerical based post-processing methods such as $\lambda_{2}$ or $\mathrm{Q}$ criteria which are used in the current study can extract three-dimensional flow structures which seem to be difficult or expensive by using experimental apparatus.

Table 2 summarizes some of the previous numerical studies, which deal with different aspects of numerical simulation of flow around flat plate. In the most broad sense, these studies can be divided into two large categories. Those that focus on the numerical simulation such as new solution methods, and those in which the focus is analysis of the flow, with numerical simulations regarded as a principal or complimentary tool for this analysis.

Here, we briefly review a few selected numerical studies, which demonstrate the increasing level of detail and complexity of the flow analysis achievable thanks to evolution of the numerical techniques over the past two decades. Lasher [25] performed two-dimensional CFD simulation of flow around a flat plate using different turbulence models [25]. Their results showed that the mean value analysis under-predicts the drag force irrespective of the turbulence model. However, the unsteady cases successfully evaluated correct values for this parameter. The Reynolds number was 32,200 based on

Table 2 A summary of previous numerical studies on flow over flat plate

\begin{tabular}{llll}
\hline Numerical Simulations & AR & Reynolds & Inclination angle \\
\hline Tamada \& Miyagi [13] & 2D & small -- $>\infty$ & $90^{\circ}$ \\
Kuwahara [14] & 2D & - & $30^{\circ}, 45^{\circ}, 60^{\circ}, 75^{\circ}, 89^{\circ}$ \\
Sarpkaya [15] & 2D & inviscid & $40^{\circ}, 50^{\circ}, 60^{\circ}, 70^{\circ}, 80^{\circ}$ \\
Hudson \& Denis [16] & 2D & $0.1 \sim 20$ & $90^{\circ}$ \\
Chein \& Chung [17] & 2D & - & $60^{\circ}, 90^{\circ}$ \\
Chua et al. [18] & 2D & 5000 & $90^{\circ}$ \\
Dennis et al. [19] & 2D & 100 & $90^{\circ}$ \\
Tamaddon-Jahromi [20] & 2D & 126,250 and 500 & $90^{\circ}$ \\
Najjar \& Vanka [21] & 2D & $80 \sim 1000$ & $90^{\circ}$ \\
Najjar \& Vanka [22] & 3D & 1000 & $90^{\circ}$ \\
Yeung \& Parkinson [23] & 2D & inviscid & $14.85^{\circ} \sim 90^{\circ}$ \\
Najjar \& Balachandar [24] & 3D & 250 & $90^{\circ}$ \\
Lasher [25] & 2D & 32,200 & 90 \\
Narasimhamurthy \& Anderson [26] & 3D & 750 & $90^{\circ}$ \\
Taira \& Colonius [27] & 3D & $300 \sim 500$ & $0^{\circ} \sim 60^{\circ}$ \\
Afgan et al. [28] & 3D & 750 & $45^{\circ}, 90^{\circ}$ \\
Yang et al. [29] & 3D & $525 \sim 1000$ & $20^{\circ}$ \\
Tian et al. [30] & 3D & 150,000 & $90^{\circ}$ \\
Hemmati et al. [31] & 3D & $1200 \sim 2400$ & $90^{\circ}$ \\
\hline
\end{tabular}


the chord length and inlet velocity. They tested different turbulence models including standard $\mathrm{k}-\varepsilon$, the extended $\mathrm{k}-\varepsilon$ developed by Chen and Kim [32], RNG k $-\varepsilon$ [33] and Speziale [34] turbulence models. Based on their evaluation, the Speziale [34] model which was anisotropic model predicted more close drag coefficients to the experimental values of Takeuchi and Okamoto [35].

Najjar and Vanka [21] used two-dimensional numerical simulation to model flow over a normal flat plate at $80<\operatorname{Re}<1000$. The numerical method was based on a fifth-order upwind scheme for the convective terms and a fourth-order scheme for diffusion term. They used a direct solver according to eigenvalue decomposition and a capacitance matrix technique for the pressure Poisson equation. They observed that the mean drag force is over-predicted by a factor of 1.6 comparing to the value reported by Fage and Johansen [1]. The difference was expected to be due to the initiation of flow three-dimensionality for Reynolds numbers larger than 200. Najjar and Vanka [22] continued their study and performed a threedimensional Direct Numerical Simulation on flow over a normal flat plate at the Reynolds number of 1000 . The mean drag obtained from the simulation showed good agreement with the experimental results. The three-dimensional case was also able to capture the regions of streamwise vorticity in the form of ribs connecting the adjacent vortex rollers. They concluded that the enhancement in the quality of the results from two to three-dimensional case is due to the introduction of threedimensional effects in the second case.

Taira and Colonius [27] using the immersed boundary method performed threedimensional numerical simulations of flow around low-aspect-ratio flat plate at Reynolds numbers of 300 and 500 with emphasis on the unsteady vortex dynamics at post-stall angles of attack. They performed an oil tow-tank experiment in order to validate their numerical simulation. They studied the effect of different parameters including the aspect ratio, angle of attack and planform geometry on the flow structure behind the plate. They noticed that the tip vortices significantly influence the vortex dynamics and stabilize the flow which has significant influence on aerodynamic forces. Based on the aspect ratio, angle of attack and Reynolds number, different stability regimes were observed including a) a stable steady state, b) a periodic unsteady state or c) an aperiodic unsteady state. This evaluation was performed by looking at the variation of angle of attack relative to aspect ratio parameter. They noticed that the steady state condition is achieved over a wide range of angles of attack with lower aspect ratios since the tip vortices are able to prevent the leading and trailing edge vortices from shedding. By increasing the aspect ratio, vortex shedding from leading and trailing edges started and a periodic behavior was observed. Although they evaluated flat plates in different flow configurations, their research was carried out at low Reynolds numbers and flow analysis for higher Reynolds numbers was missing.

Tian et al. [30] performed LES to model flow over a normal flat plate with the thickness to height ratio of 0.02 at the Reynolds number of 150,000. The influence of the shape of the corners was investigated in their study. Two round corners with different curvature radii $(r=0.01 \mathrm{H}$ and $0.005 \mathrm{H})$ were modeled. The sharper corner $(r=0.005 \mathrm{H})$ case increased the mean drag, fluctuations of drag and lift forces as well as the kinetic energy in the near wake. 
Due to the significant resemblance of the flow over flat plate with flow over structures such as solar panels, analysis of flow past flat plate at different inclination angles has gained significant attention by researchers in solar panel field. The main objective of these studies is the analysis of flow over solar panels in atmospheric boundary layer and its influence on the wind loading. Basically researchers in the field of solar panel use the flat plate experiments as a benchmarking criterion for their studies. Shademan and Hangan [36, 37] performed RANS studies on a stand-alone solar panel immersed in atmospheric boundary layer at different inclination angles and azimuthal wind directions to distinguish the critical wind loading conditions. No significant change was observed for the inclination angles analyzed. However, they noticed that for the conditions at which the wind is perpendicular either to the front or back face, the panel experiences the maximum wind loading. Although their analysis presented important information for solar panel industry, it did not include any peak value results which require unsteady simulation. Further to this study, Shademan et al. [38] carried out another RANS study on the effect of gap spacing between individual panels and different ground clearance on the wind loading of the solar panels. For the gap spacing they modeled, no significant change was noticed for the experienced wind loading. They also observed that increasing the ground clearance has a large influence on the increase of the wind loading. They concluded this as a result of higher shear flow interaction separated from the bottom and top edges of the panel in higher ground clearance cases. Due to the important effect of ground clearance on the generation of unsteady wind fluctuations, Shademan et al. [39] performed Detached Eddy Simulation (DES) on solar panels with different ground clearance. They noticed that by increasing the ground clearance, the mean and peak wind loading increases. They also found an optimum ground clearance for solar panels at which they experience the least wind loading.

According to the literature, there are various types of experiments (Table 1) and numerical simulations (Table 2) on flow over flat plate. In the experimental side, most of these studies are reporting the mean value results and in numerical side are dealing with modeling issues. It is observed that a comprehensive document which evaluates different aspects of flow over flow plate including the aspect ratio and inclination angle and their effects on different flow parameters such as shedding frequency and aerodynamic loading is missing.

Therefore, in the current study, it is of interest to investigate the effect of different aspect ratios and inclination angles on the mean and unsteady flow parameters. The results of this research can help to a better understanding of the trend of the unsteady aerodynamic loading of flat plate in different configurations. Due to the resemblance of the flow field with flow over solar panels [36-39], the results of current study can be used as guidance for the design of these kinds of structures.

To achieve the discussed objectives, the manuscript is formed as following: Section 2 provides details of the numerical method including the geometry modeling, governing equations and boundary conditions. In section 3 which is the benchmarking part, the numerical setup used in the current analysis is evaluated by comparing the results with the available experimental data. Finally, section 4 presents the analysis of the results and discussions. 


\section{Numerical method}

\subsection{Geometry modeling and boundary conditions}

The geometry of flat plate modeled in the current study is similar to the one tested by Fage and Johansen [1] which has a chord length of $0.15 \mathrm{~m}$. The front surface of the plate is flat while the other is slightly tapered from the center (Fig. 2b). The geometry is simulated at three different inclination angles including $\alpha=30^{\circ}, 60^{\circ}$ and $90^{\circ}$ for three aspect ratios $(\mathrm{S} / \mathrm{C})$ of $0.5,2$ and 5 , where $S$ is the span and $C$ is the chord length of the plate. According to the recommendations by Bosch and Rodi [40], the domain dimensions are chosen in a way to have the negligible blockage effect on the plate at above mentioned configurations. The computational domain is taken to extend $20 \mathrm{~S}$ in the downstream direction and $5 \mathrm{~S}$ in vertical, transverse and upstream directions from the plate, as illustrated in Fig. 2a. Based on these domain dimensions, the maximum blockage in all cases is about $1 \%$, which is much smaller than the $3 \%$ maximum value suggested by Franke et al. [41].

The two sides (left and right), top and bottom boundaries were set to have slip condition. This is identical to a far field free stream surface. The plate surface is considered to be a solid wall with no-slip, no penetration conditions. A uniform air velocity of $15(\mathrm{~m} / \mathrm{s})$ is applied at the inlet of the domain, which is similar to the air velocity considered by Fage and Johansen [1] in their experiments. The corresponding Reynolds number is 150,000 based on the chord length of the plate and incoming air velocity. According to the length of the downstream region (20S), it is expected to have a fully developed flow at the exit of the domain. A ZeroGradient boundary condition which imposes zero changes for the flow parameters is used at the exit boundary. A fully structured mesh scheme was generated for the domain including hexahedral elements in all three directions. In order to capture the high velocity gradients, a finer mesh was considered for the wall proximity region in three directions. The LES mesh study criterion, recommended by Piomelli and Chasnov [42] $\left(\Delta \mathrm{x}^{+} \cong 100, \mathrm{y}^{+} \cong 1\right.$ and $\left.\Delta \mathrm{z}^{+} \cong 30\right)$ is satisfied for regions of interest close to the plate $(-1<\mathrm{x} / \mathrm{C}<1,-1<\mathrm{y} / \mathrm{C}<1$ and $-1<\mathrm{z} / \mathrm{C}<1)$. In these equations, $\Delta \mathrm{x}^{+}, \Delta \mathrm{z}^{+}$ and $\mathrm{y}^{+}$are non-dimensional numbers $\left(\Delta \mathrm{x}^{+}=\Delta \mathrm{xu}_{\mathrm{\tau}} / \mathrm{v}, \Delta \mathrm{z}^{+}=\Delta \mathrm{zu}_{\mathrm{\tau}} / \mathrm{v}, \mathrm{y}^{+}=\mathrm{u}_{\mathrm{t}} \mathrm{y} / \mathrm{v}\right)$, where $\mathrm{u}_{\mathrm{\tau}}$ is the friction velocity, $y$ is the normal distance from the wall and $v$ is the kinematic viscosity. Three inclination angles were modeled for each aspect ratio, which results in a total number of nine simulation cases. Details of the computational domain and mesh generated for each case are shown in Table 3.

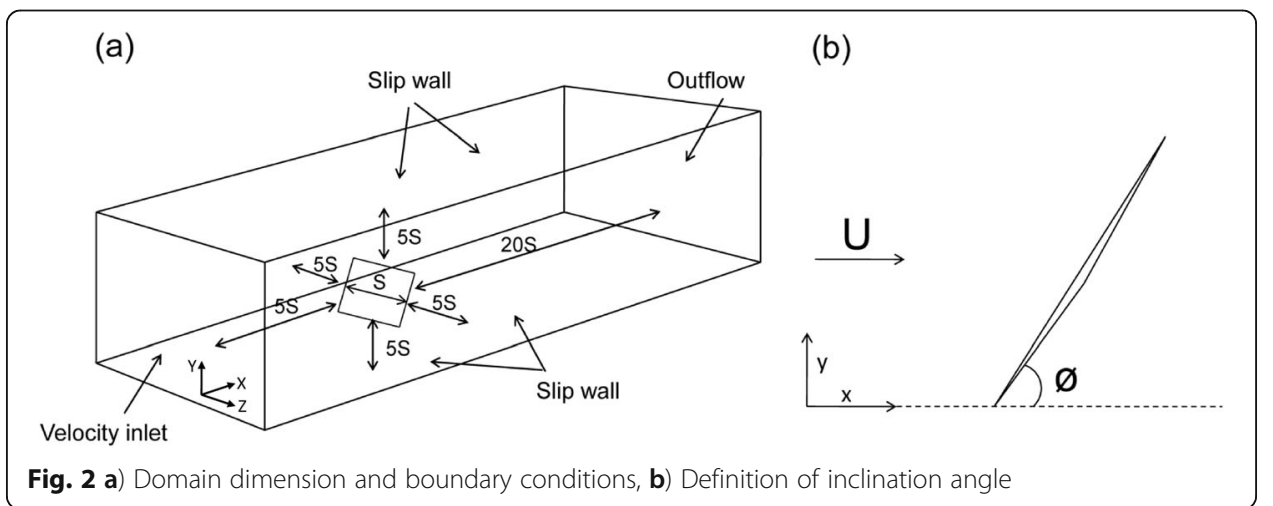


Table 3 Details of the computational domain and mesh

\begin{tabular}{llllll}
\hline $\begin{array}{l}\text { Aspect } \\
\text { Ratio }\end{array}$ & $\begin{array}{l}\text { Inclination } \\
\text { angle }\left(\mathrm{a}^{\circ}\right)\end{array}$ & $\begin{array}{l}\text { Upstream } \\
\text { length }(\mathrm{m})\end{array}$ & $\begin{array}{l}\text { Downstream } \\
\text { length }(\mathrm{m})\end{array}$ & $\begin{array}{l}\text { Transverse direction length }(\mathrm{m}) \text { from } \\
\text { the plate in each side }\end{array}$ & $\begin{array}{l}\text { Number } \\
\text { of cells }\end{array}$ \\
\hline 0.5 & $30,60,90$ & 0.375 & 1.5 & 0.375 & $4,710,000$ \\
2 & $30,60,90$ & 1.5 & 6 & 1.5 & $6,368,250$ \\
5 & $30,60,90$ & 3.75 & 15 & 3.75 & $7,505,050$ \\
\hline
\end{tabular}

\subsection{Governing equations}

The governing equations used in LES are obtained by filtering the unsteady NavierStokes equations in Fourier space. The filtered equations are as follows:

$$
\begin{aligned}
& \frac{\partial}{\partial \mathrm{x}_{\mathrm{i}}}\left(\overline{\mathrm{u}}_{\mathrm{i}}\right)=0 \\
& \frac{\partial}{\partial \mathrm{t}}\left(\overline{\mathrm{u}}_{\mathrm{i}}\right)+\frac{\partial}{\partial \mathrm{x}_{\mathrm{j}}}\left(\overline{\mathrm{u}}_{\mathrm{i}} \overline{\mathrm{u}}_{\mathrm{j}}\right)=\frac{\partial}{\partial \mathrm{x}_{\mathrm{j}}}\left(v \frac{\partial \sigma_{\mathrm{ij}}}{\partial \mathrm{x}_{\mathrm{j}}}\right)-\frac{1}{\rho} \frac{\partial \overline{\mathrm{p}}}{\partial \mathrm{x}_{\mathrm{i}}}-\frac{1}{\rho} \frac{\partial \mathrm{\tau}_{\mathrm{ij}}}{\partial \mathrm{x}_{\mathrm{j}}}
\end{aligned}
$$

where $\mathrm{i}$ indicates the spatial dimension, $\mathrm{p}$ is the pressure, $\rho$ is the density, $v$ is the kinematic viscosity, $\bar{u}_{i}$ is the resolved velocity field and $\tau_{i j}$ is the subgrid scale stress defined by:

$$
\tau_{i j}=\rho \overline{u_{i} u_{j}}-\rho \overline{u_{i} u_{j}}
$$

The subgrid-scale stresses resulting from the filtering operation are unknown, and require modelling. In the current simulations, the dynamic Smagorinsky method [43] is used for modelling the subgrid-scale stresses.

The finite volume method is used to discretize the governing equations. Central differencing scheme was used for discretizing the convective terms. A fully implicit second order method was used for time marching. In order to maintain the courant number less than one, the time step is kept at $1 \times 10^{-5}(\mathrm{~s})$. For coupling the velocity and pressure, the SIMPLE algorithm was implemented. The OpenFOAM (2.2.3) source code [44] was used for solving the governing equations. The mean value flow parameters are obtained by averaging the unsteady results long after the flow has reached a fully established condition. During the simulations, the drag force exerted on the plate was monitored, and the solution was considered converged when no significant change in the repetitive behavior of the drag force in time was observed. For all results presented in

this paper, the continuity and momentum equation residuals are all of the order of $10^{-5}$.

\subsection{Validation of the numerical model}

In order to evaluate the accuracy of the numerical model, the experiments carried out by Fage and Johansen [1] on flow past flat plate were modeled at different inclination angles. In their experiments, the flat plate was mounted vertically with a small gap between its ends and the floor and roof of the wind tunnel. Different flow parameters including pressure distribution around the plate and Strouhal number were captured at different inclination angles.

Large Eddy Simulations (LES) were performed to model the flow past flat plate at different inclination angles including $\alpha=30^{\circ}, 50^{\circ}, 90^{\circ}$. The numerical simulation was designed in a way to mimic the experimental setup carried out by Fage and 
Johansen [1]. The plate modeled has a span of $1.5 \mathrm{~m}$ and a chord length of $0.15 \mathrm{~m}$. The cross section of the flat plate modeled is presented in Fig. 2b. The recommendations by Bosch and Rodi [40] were used to define the domain dimensions in order to reduce blockage issues. Slip condition (zero shear stress) was applied on top, bottom and two side boundaries, and for the plate, a wall boundary condition was used. A uniform velocity of $15(\mathrm{~m} / \mathrm{s})$ corresponding to a Reynolds number of $1.5 \times 10^{5}$ based on the chord length of the plate was applied at the inlet. Note that, the LES mesh requirement criterion [42] was also applied in this section. A fully structured mesh scheme was generated for the domain with a total of $5.9 \times 10^{6}$ cells. Temporal averaging of the unsteady results was performed over a large number of shedding periods $(\sim 20)$.

A comparison between the mean pressure coefficient $\left(\mathrm{C}_{\mathrm{P}}=\left(\mathrm{P}-\mathrm{P}_{\infty}\right) /\left(0.5 \rho_{\infty} \mathrm{U}_{\infty}^{2}\right)\right)$ obtained from current simulations and the experiments by Fage and Johansen [1] are presented in Fig. 3a, c, e. Good agreement observed between the two sets of results confirms the validity of the model for the analysis of mean flow over flat plate at different configurations.

Figures $3 \mathrm{~b}, \mathrm{~d}, \mathrm{f}$ visualize the mean pressure coefficient contours for $\alpha=30^{\circ}, 50^{\circ}$ and $90^{\circ}$, respectively. These figures show that the size of the recirculation region behind the plate increases as the inclination angle increases. The size of the region was observed to extend to $\mathrm{x} / \mathrm{C}=1.5$ for $\alpha=30^{\circ}, \mathrm{x} / \mathrm{C}=1.7$ for $\alpha=50^{\circ}$ and $\mathrm{x} / \mathrm{C}=3$ for $\alpha=90^{\circ}$. By increasing the inclination angle to $\alpha=90^{\circ}$, the temporally averaged shape of the recirculation region becomes symmetric and the pressure difference reaches the maximum value relative to $\alpha=30^{\circ}$ and $50^{\circ}$ cases.

To further evaluate the accuracy of the numerical model in temporal space, the vortex shedding frequency determined from current simulations at different inclination angles were compared with the available experimental results. In this regard, Fast Fourier Transform (FFT) was performed on the time history of aerodynamic forces of the plate. The Strouhal numbers $\left(\mathrm{fc}_{\mathrm{c}} / \mathrm{U}_{\infty}\right)$ calculated based on the peak frequency (f) as captured from the power spectra graphs (Figs. 4a-c), chord length (c) and approaching free stream velocity $\left(U_{\infty}\right)$ are summarized in Table 4.

Good agreement can be observed between the results obtained from current LES and experiments for $30^{\circ}$ and $50^{\circ}$ inclination angles. A marginal difference of $7 \%$ can be seen between the results of LES and experiments for $90^{\circ}$ case. This evaluation confirms the accuracy of the numerical model for the unsteady analysis of flow over flat plate. The analysis discussed herein provides guidelines to expand the numerical simulation to model the flow over flat plates with different aspect ratios at different inclination angles, as described in the following chapters.

\section{Mean flow results (effect of aspect ratio and inclination angle)}

\subsection{Mean aerodynamic forces}

In this section, it is of interest to investigate the behaviour of mean aerodynamic forces with different aspect ratios and inclination angles. In this regard, the variation of drag and lift coefficients $\left(C_{D}, C_{L}\right)$ for different aspect ratios $(0.5,2$ and 5$)$ and inclination 


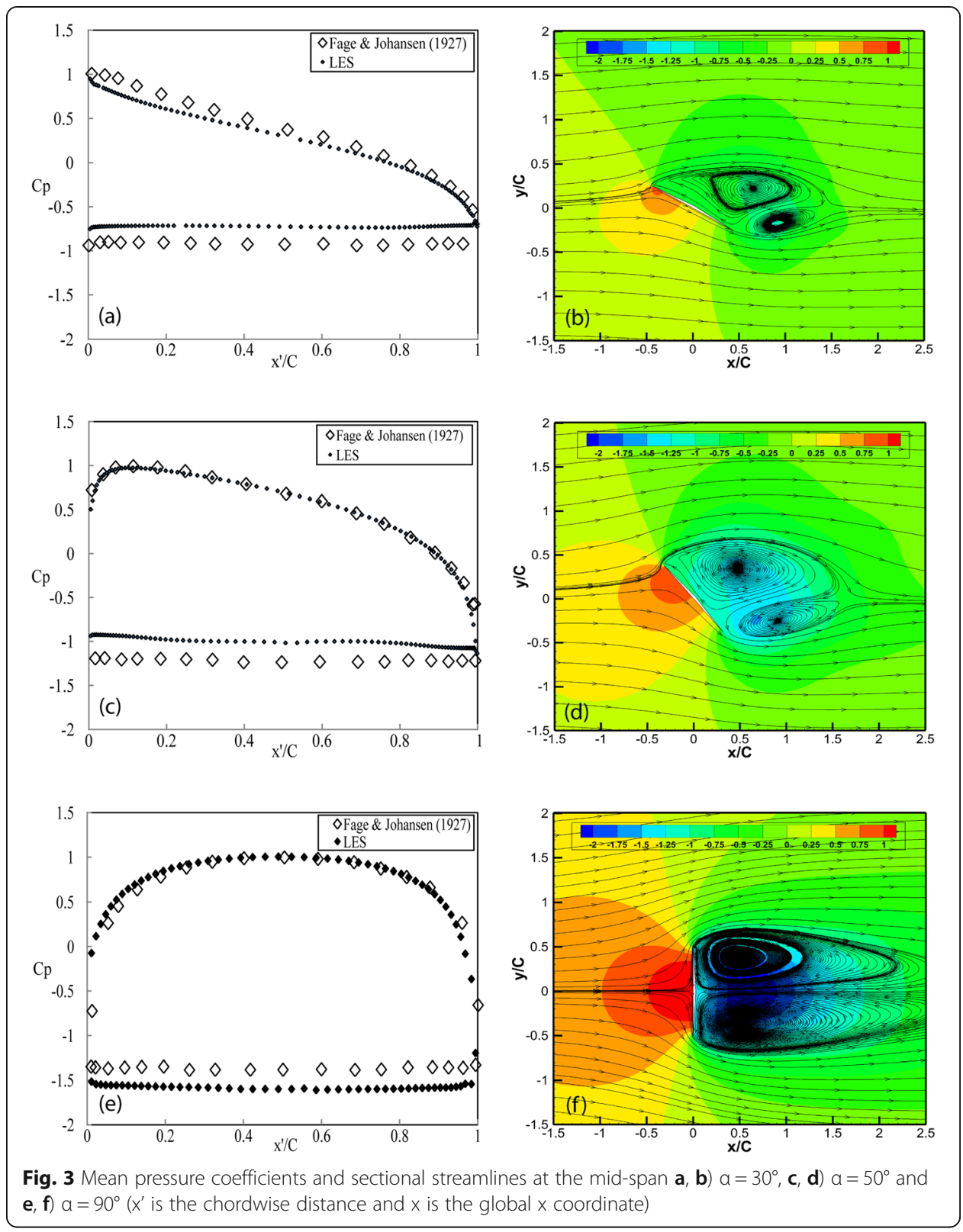

angles $\left(30^{\circ}, 60^{\circ}\right.$ and $\left.90^{\circ}\right)$ are presented in Figs. $5 \mathrm{a}, \mathrm{b}$ and c, d respectively. The aerodynamic force coefficients can be determined using the following equations:

$$
\begin{aligned}
& C_{D}=\frac{F_{D}}{0.5 \rho U_{\infty}^{2} A} \\
& C_{L}=\frac{F_{L}}{0.5 \rho U_{\infty}^{2} A}
\end{aligned}
$$

where, $F_{D}$ and $F_{L}$ are drag and lift forces, respectively, $\rho$ is density, $A$ is the area of the plate facing the flow stream (projected frontal area) and $U_{\infty}$ is the free stream velocity.

Figures $5 \mathrm{a}$, b show the variation of drag and lift coefficients with inclinations angle at different aspect ratios. These coefficients were obtained after performing the averaging 

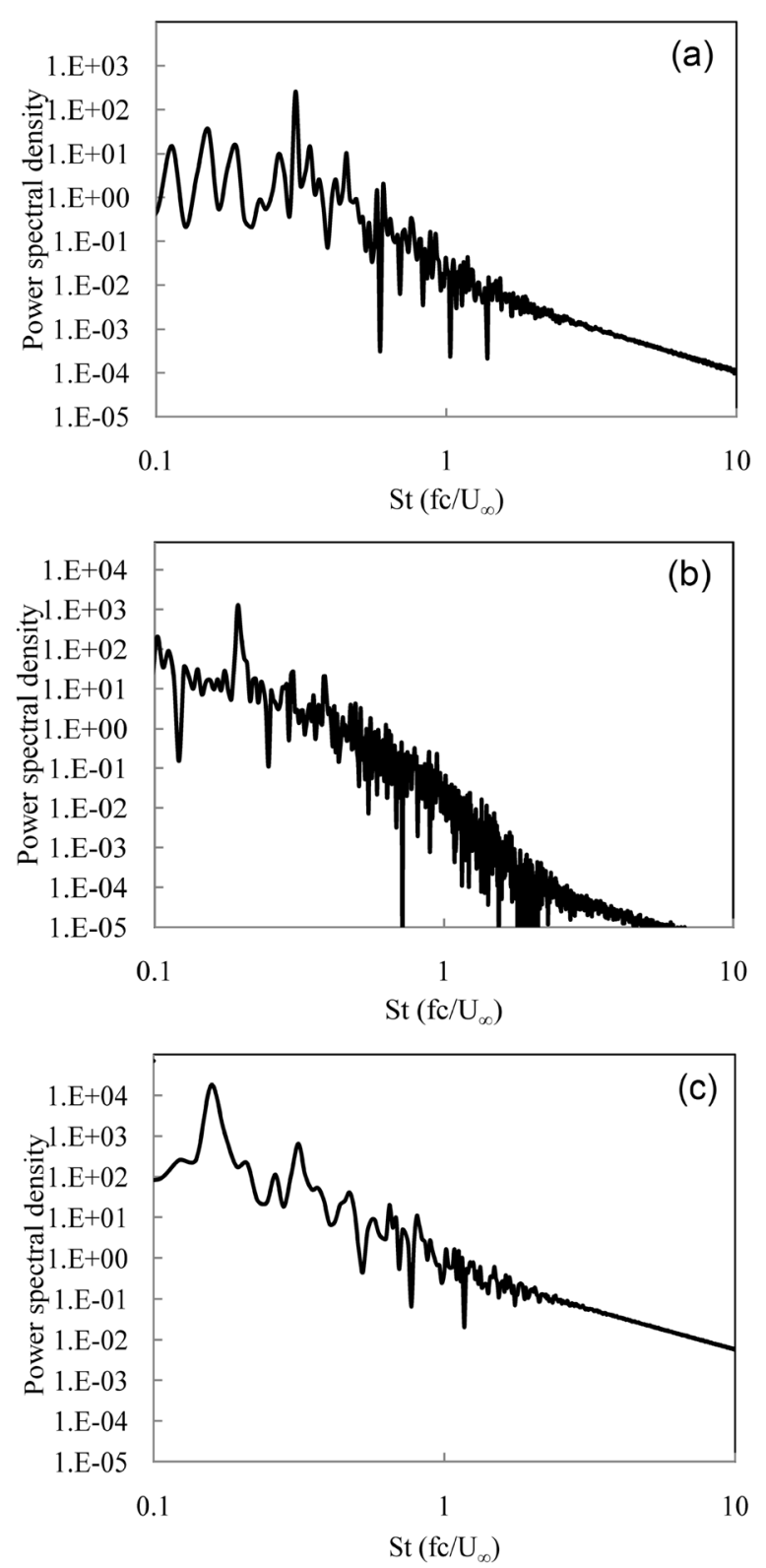

Fig. 4 Power spectra at a) $a=30^{\circ}$, b) $a=50^{\circ}$, c) $a=90^{\circ}$

Table 4 Strouhal number comparison obtained from experiments and current LES

\begin{tabular}{lll}
\hline $\begin{array}{ll}\text { Inclination } \\
\text { angle (a) }\end{array}$ & Strouhal number & \\
\cline { 2 - 3 } & Fage and Johansen [1] & Current LES \\
\hline $30^{\circ}$ & 0.307 & 0.301 \\
$50^{\circ}$ & 0.196 & 0.194 \\
$90^{\circ}$ & 0.146 & 0.157 \\
\hline
\end{tabular}



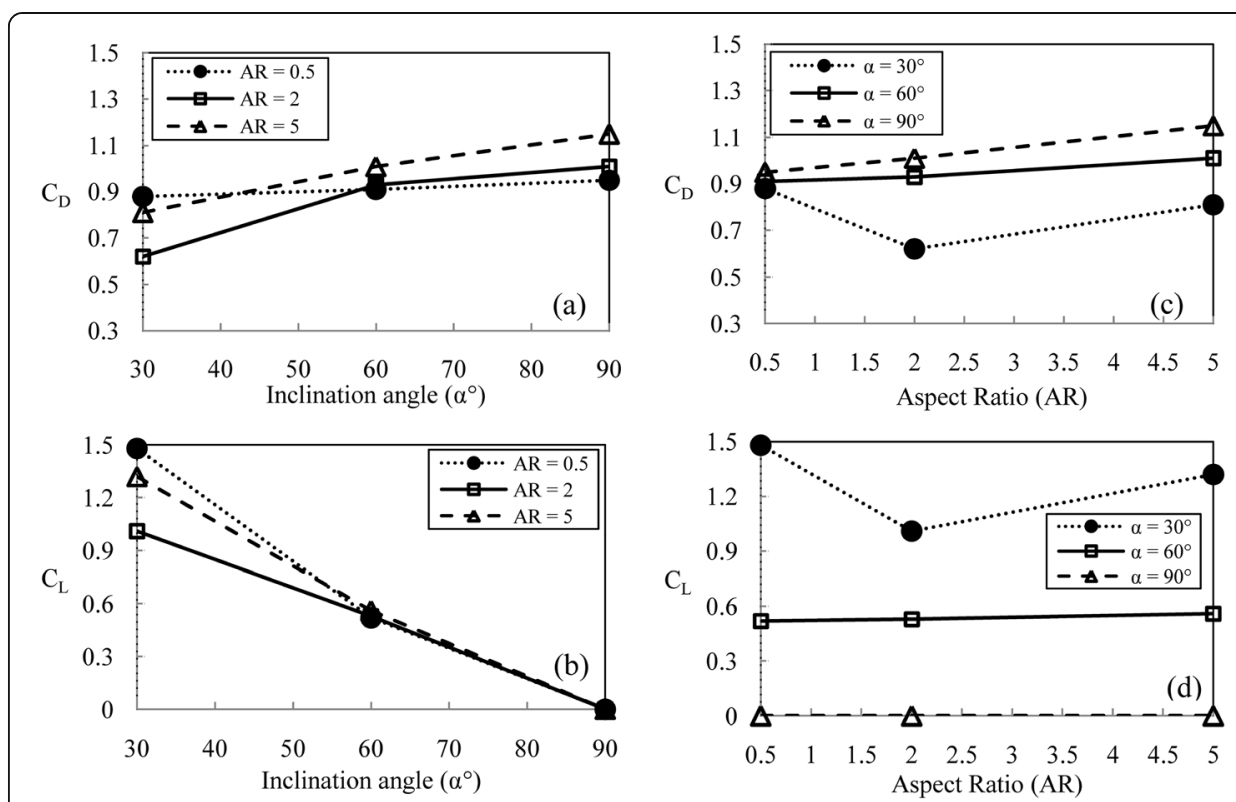

Fig. 5 Variation of $C_{D}$ and $C_{L}$ with $\mathbf{a}, \mathbf{b}$ ) inclination angle and $\mathbf{c}, \mathbf{d}$ ) aspect ratio

process over a large number of time steps on the unsteady values. The results show that in general, increasing the inclination angle, increases the drag and reduces the lift irrespective of the aspect ratio. However, higher aspect ratios are associated with larger drag changes when the inclination angle increases from $30^{\circ}$ to $90^{\circ}$. An opposite trend is observed for lift coefficient where, bigger changes occur for smaller aspect ratios when moving from $30^{\circ}$ to $90^{\circ}$ inclinations angles. The comparison of the results for $30^{\circ}<\alpha<$ $60^{\circ}$ and $60^{\circ}<\alpha<90^{\circ}$ also shows that the increase of the drag and reduction of the lift are larger for $30^{\circ}<\alpha<60^{\circ}$.

The variation of the drag and lift forces with the aspect ratio at different inclination angles are presented in Figs. 5c, d. An increasing trend can be noticed for drag when the aspect ratio increases for $60^{\circ}$ and $90^{\circ}$ inclination angles. However, lift coefficient does not show significant change for these two inclination angles. An irregular trend was observed for $\alpha=30^{\circ}$, where the drag and lift coefficients show larger values at $A R=$ 0.5 relative to other aspect ratios. From $A R=0.5$ to $A R=2$, a reducing trend can be seen for both drag and lift coefficients for this specific inclination angle $\left(\alpha=30^{\circ}\right)$, however, for $\mathrm{AR}=2$ to $\mathrm{AR}=5$, an increasing trend was observed. This phenomenon might be due to the influence of the flow structures in the wake region which causes larger pressure difference between windward and leeward faces of the plate for this specific case. The mean pressure distribution curves presented in the next section present more details regarding this phenomenon.

\subsection{Mean pressure distribution}

In order to investigate the variation of the mean lift and drag coefficients with the aspect ratio and inclination angle, it is possible to look at the pressure changes in the windward and leeward surfaces of the mid-plane. 
Figures 6a-c show mean pressure coefficients for different aspect ratios including $\mathrm{AR}=0.5,2$ and 5 at three inclination angles of $\alpha=30^{\circ}, 60^{\circ}$ and $90^{\circ}$ extracted at $\mathrm{x}-\mathrm{y}$ mid-plane of the plate.

Figure 6a shows that at $\alpha=30^{\circ}$, increasing the aspect ratio from 2 to 5 results in marginal reduction in the wake pressure which is associated with small increase in
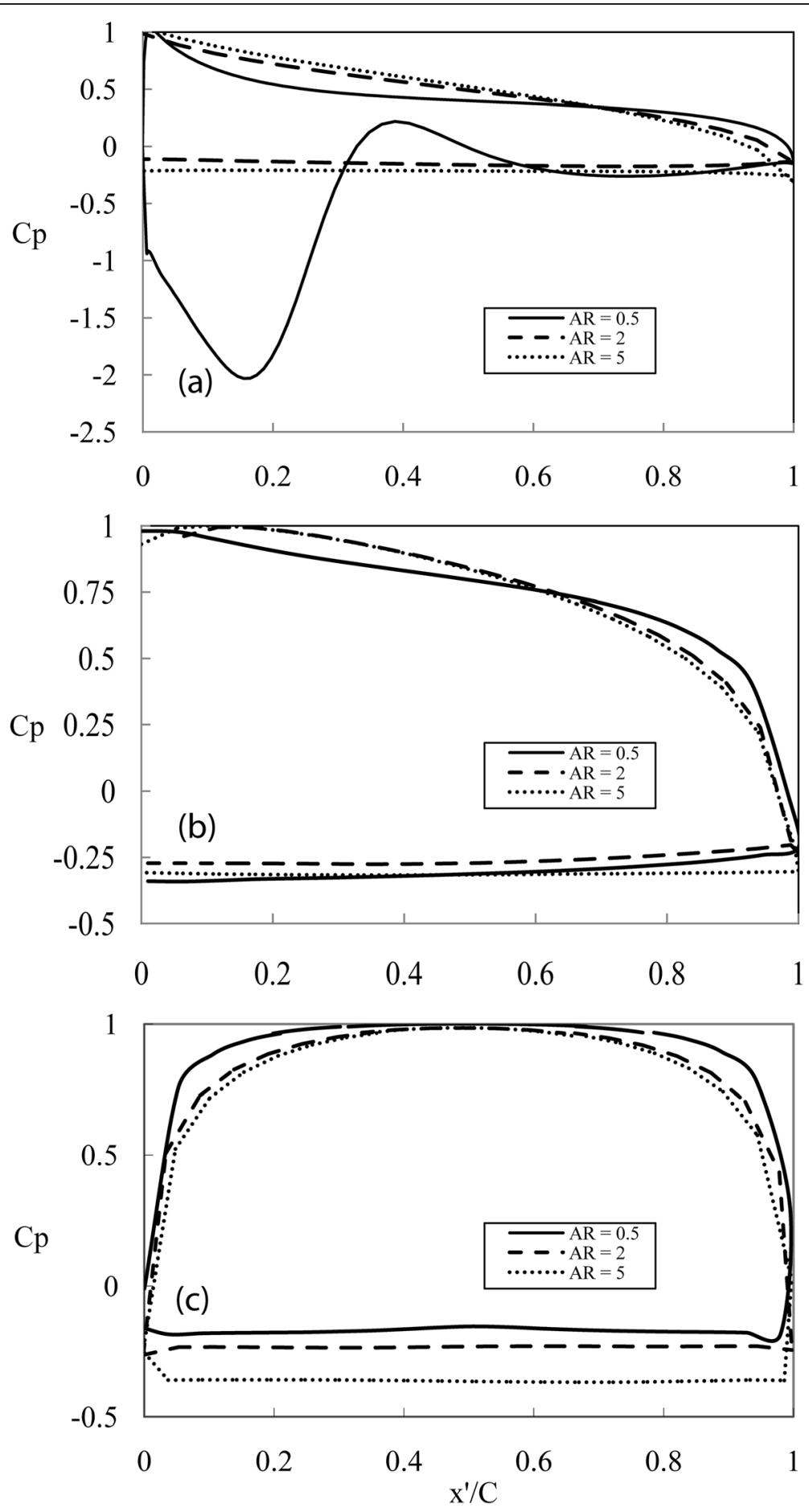

Fig. 6 Pressure coefficient $\left(C_{p}\right)$ for different aspect ratios at a) $\left.\left.a=30^{\circ}, \mathbf{b}\right) a=60^{\circ}, \mathbf{c}\right) a=90^{\circ}$ 
the aerodynamic loading on the plate. This is associated with the increase observed in drag coefficient for $\alpha=30^{\circ}$ when moving from $A R=2$ to $A R=5$ as shown in Fig. 5c. At $A R=0.5$, a large reduction in the wake pressure is observed particularly in $0<\mathrm{x} / \mathrm{C}<0.3$ relative to other aspect ratios. This is associated with higher aerodynamic loading for this specific case.

At $\alpha=60^{\circ}$ (Fig. 6b), no significant change in Cp distributions is noticed when aspect ratio increases from 0.5 to 5 . This confirms that increasing the aspect ratio from 0.5 to 5 is not expected to cause a significant change in the aerodynamic loading for this inclination angle. This is another interpretation for the trend observed in Figs. 5c, d where almost no change was observed for the drag and lift coefficients when moving from $A R=0.5$ to $A R=5$ at $\alpha=60^{\circ}$. At $\alpha=90^{\circ}$ (Fig. 6c), increasing the aspect ratio from 0.5 to 5 reduces the pressure coefficient at the proximity of the two edges of windward side of the plate. For the leeward side, a reduction in pressure coefficient is observed when aspect ratio increases from 0.5 to 5 . The influence of this behavior was seen on mean drag and lift coefficients (Figs. 5c, d) where moving from $A R=0.5$ to 5 at $\alpha=90^{\circ}$ resulted in slight increase for drag and no change for lift.

Note that, the analysis presented in this study is limited to aspect ratios between 0.5 and 5, however comparison of the results with the infinite span cases (Fig. 3a, c, e) proves existence of much larger pressure differences between front and back surfaces of the infinite span cases compared to smaller aspect ratio cases.

In order to evaluate the pressure coefficients in mid-sections of each case, Fig. 7a-i are presented which show the mean pressure coefficient $\left(C_{p}\right)$ contours and sectional streamlines at different inclination angles and aspect ratios plotted at $\mathrm{x}-\mathrm{y}$ mid-plane. The irregular behaviour of the pressure coefficient at $\alpha=30^{\circ}$ and $A R=0.5$ (Fig. 6a) can be explained by examining Fig. 7a. According to Fig. 7a, there is a vortex close to the top corner in the leeward face which results in a lower pressure relative to other regions of the back surface. In this particular case, separation does not dominate the entire back surface and flow reattaches after separation. This was seen in Fig. 6a where the low pressure curve was present only for a very small region of the back surface, and for other parts, it was close to the level of the other aspect ratio cases. By increasing the inclination angle to $\alpha=60^{\circ}$ and $90^{\circ}$ while keeping the aspect ratio constant, the distance of the vortices from the leeward surface increases in the wake region (Figs. 7b, c). Although a bigger wake region is observed for these two cases compared to $\alpha=30^{\circ}$, they show more positive pressures $(-0.5<\mathrm{Cp}<0)$ compared to $\alpha=30^{\circ}$ case where $1<\mathrm{Cp}<-1.5$. For the frontal area (windward surface), the $\mathrm{Cp}$ varies between $0<\mathrm{Cp}<1$ for all cases at this specific aspect ratio.

At $\mathrm{AR}=2$ and 5 and for similar inclination angles, the flow pattern in the wake region is very close to each other with minor differences in the length of the recirculation regions. Figure $7 \mathrm{~g}$ shows at $\mathrm{AR}=5$, the vortices are closer to the plate relative to $\mathrm{AR}=2$ (Fig. $7 \mathrm{~d}$ ). By moving to $\alpha=60^{\circ}$, the recirculation region expands and becomes elongated towards the downstream (Figs. 7e, h). At $\alpha=90^{\circ}$, the recirculation regions reach their maximum length relative to the previous cases and follow a symmetrical behvaiour (Figs. 7f, i). A maximum recirculation length of $\mathrm{x} / \mathrm{C}=2.5$ was observed for $\mathrm{AR}=0.5, \mathrm{x} / \mathrm{C}=4$ for $\mathrm{AR}=2$ and $\mathrm{x} / \mathrm{C}=5$ for $\mathrm{AR}=5$ at $\alpha=90^{\circ}$. 


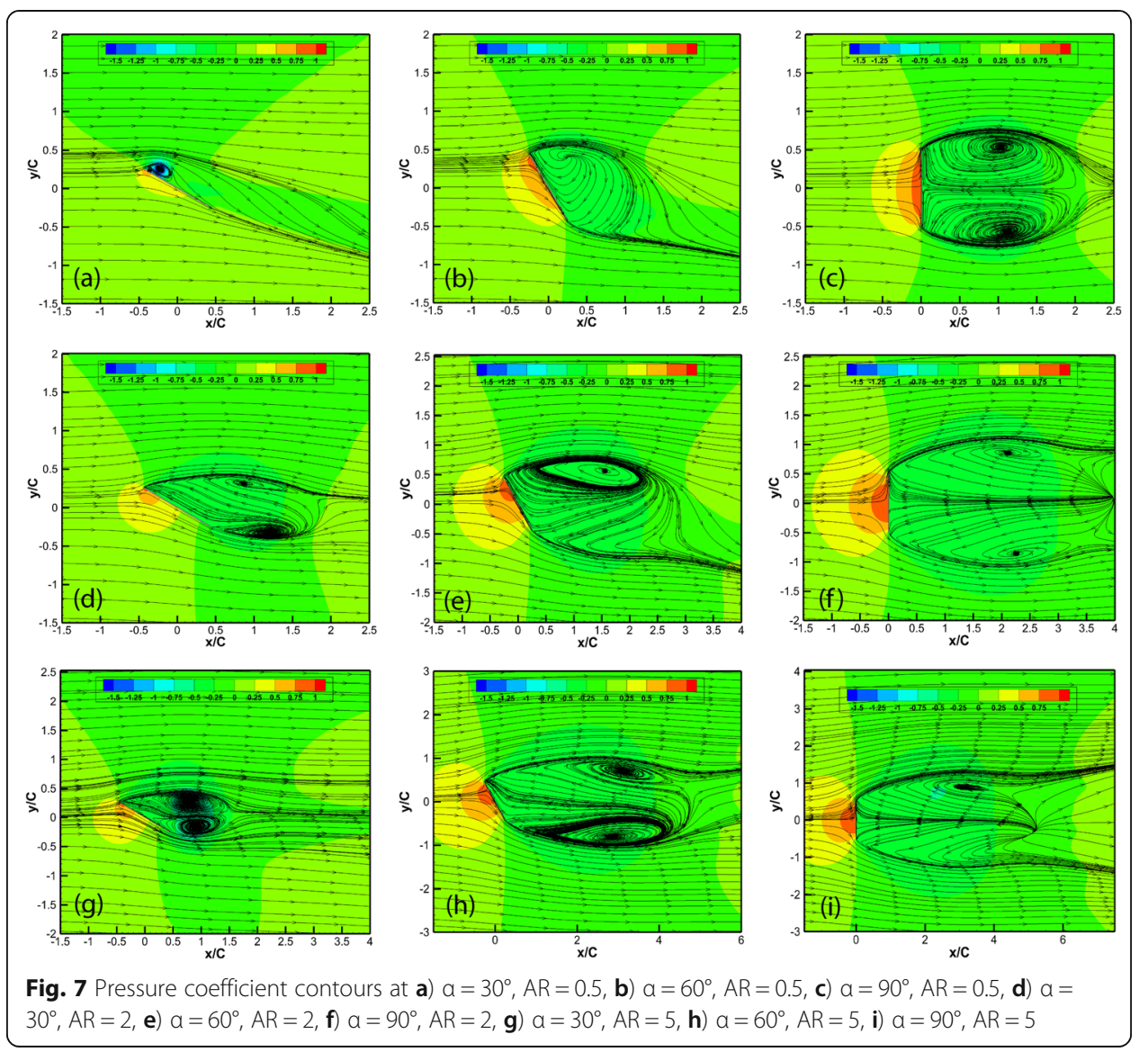

According to Fig. $7 \mathrm{a}\left(\mathrm{AR}=0.5, \alpha=30^{\circ}\right)$, the $\mathrm{Cp}$ values in the wake region at the proximity of the top edge are close to -1.5 which is significantly lower than $C p$ values in the leeward region of the other cases. Three-dimensional flow structure visualization can help to a better understanding of the phenomena occurring in this case (Fig. 7a). In this regard, the $\lambda_{2}$ criterion is used to visualize the flow structures in different flow configurations in the next section.

\subsection{Mean flow structures}

Different visualization methods can be employed to demonstrate the three-dimensional flow structures behind the plate at different aspect ratios and inclination angles. Jeong and Hussain [45] developed the $\lambda_{2}$ criterion which can be used to identify the core of the vortices in the flow field, based on the fact that these cores are related to the locations of minimum pressure in the flow. They derived the $\lambda_{2}$ criterion by calculating the gradient of the Navier-Stokes equation and decomposing the acceleration gradient term into symmetric and antisymmetric parts, expressed as $\mathrm{S}_{\mathrm{ij}} \mathrm{S}_{\mathrm{ij}}+\Omega_{\mathrm{ij}} \Omega_{\mathrm{ij}}$, where $\mathrm{S}_{\mathrm{ij}}$ and $\Omega_{\mathrm{ij}}$ are the symmetric and antisymmetric parts of the velocity gradient tensor, respectively. The Hessian of the pressure can then be connected to the vortical motions in the flow. According to the theory of multivariable calculus, the $\mathrm{S}_{\mathrm{ij}} \mathrm{S}_{\mathrm{ij}}+\Omega_{\mathrm{ij}} \Omega_{\mathrm{ij}}$ tensor has three real eigenvalues $\left(\lambda_{1} \geq \lambda_{2} \geq \lambda_{3}\right)$. The point of local pressure minimum requires two 
eigenvalues of this tensor to be negative. $\lambda_{2}$, which corresponds to the second largest eigenvalue of this tensor, is representative of the local pressure minima region. In the current study, the iso-surfaces of $\lambda_{2}$ are used for visualizing mean and instantaneous vortices in the flow.

The pressure curves and contours presented in Figs. 6 and 7 are extracted at the mid-plane and do not present any information about the three-dimensional flow pattern across the entire span. Therefore, fig. 8a-i are presented to demonstrate the threedimensional mean flow structures at different aspect ratios and inclination angles (cases discussed in Figs. 6 and 7) by using $\lambda_{2}$ iso-surfaces colored by streamwise vorticity. By visualizing the three-dimensional structures, it is possible to find a more comprehensive explanation for the pressure distribution in the wake region and consequently the resulting aerodynamic forces. Coloring the $\lambda_{2}$ iso-surfaces by streamwise vorticity also helps to specify the direction of the vortcies separated from edges and corners of the plate.

Figures 8a-c show the flow structures at $\mathrm{AR}=0.5$ and $\alpha=30^{\circ}, 60^{\circ}, 90^{\circ}$, respectively. According to Fig. 8a, due to having a short span, the two tip vortices are suppressing the expansion of the bubble behind the plate separated from the leading edge. Therefore, the separation bubble becomes significantly smaller in length and pressure magnitude comparing to other cases. The vorticity contours show opposite senses of rotation for the two tip vortices.

At $\mathrm{AR}=0.5$ and $\alpha=60^{\circ}$, the influence of tip vortices on flow separation from the top and bottom edges is reduced. A larger separation bubble in length is observed comparing to $\alpha=30^{\circ}$ and $A R=0.5$ case. This is due to the presence of the two tip vortices (with opposite senses of rotation) which have become smaller and got closer to the bottom corners of the plate. At $A R=0.5$ and $\alpha=90^{\circ}$, due to the

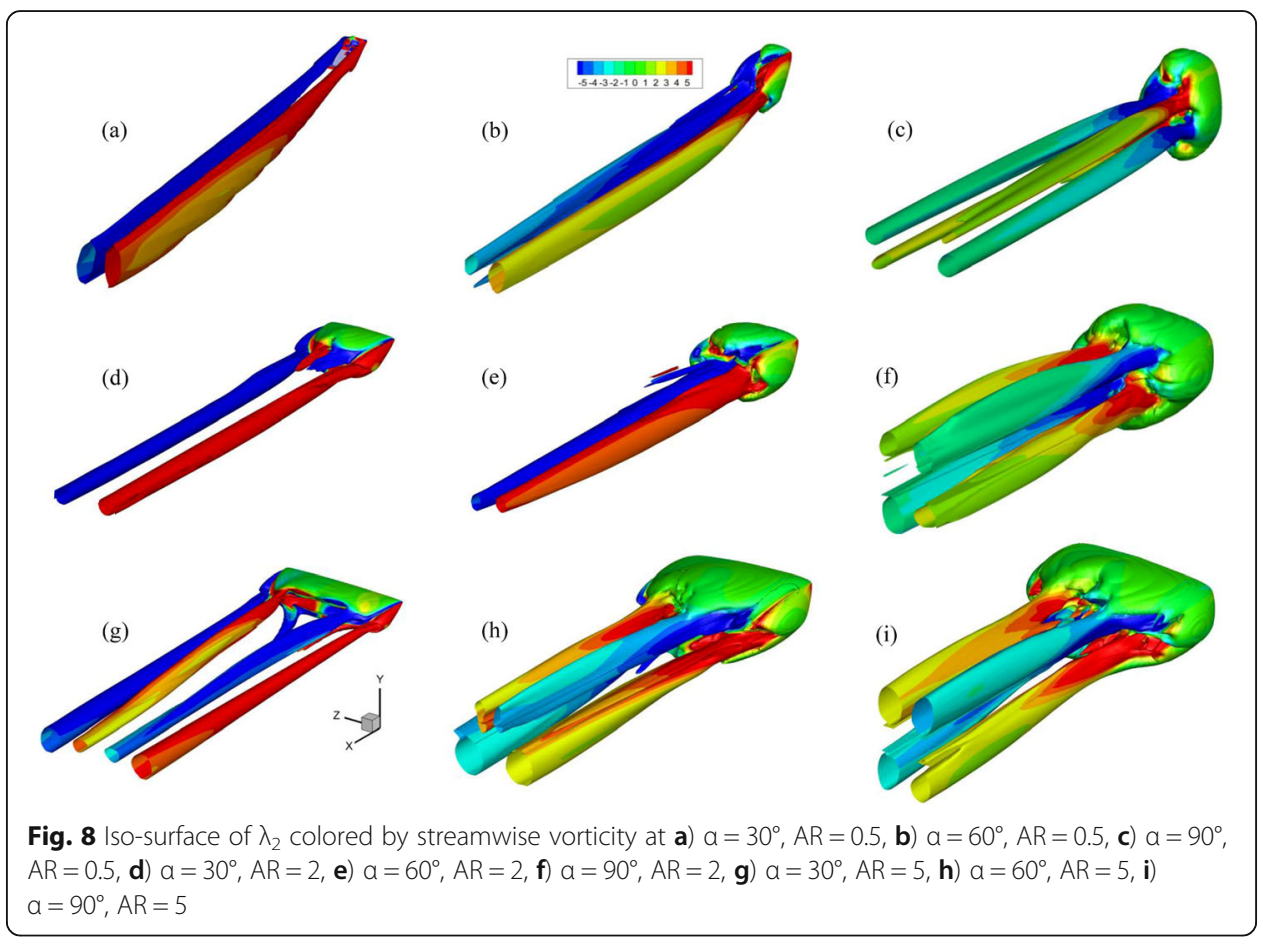


symmetrical position of the geometry with respect to the flow (comparing to $\alpha=$ $30^{\circ}$ and $\alpha=60^{\circ}$ cases), four vortices are generated which is different from the previous cases where there was only two vortices. This is because of the identical influence of the four corners in generation of the vortices. Note that, due to the inclination of the plate in $\alpha=30^{\circ}$ and $\alpha=60^{\circ}$, only the top two corners were the cause for the generation of the vortices. For this case, flow structures in the wake region show symmetrical pattern in $x-y$ and $x-z$ planes. The tip vortices along the diagonal show similar rotational directions.

At $\mathrm{AR}=2$, due to longer span comparing to $\mathrm{AR}=0.5$, the tip vortices have less influence on suppressing the separating flow from the top and bottom edges of the plate. This causes bigger separation bubbles comparing to the ones observed at $\mathrm{AR}=0.5$. Similar to the flow pattern observed at $A R=0.5$, two vortices are generated at $\alpha=30^{\circ}$ and $\alpha=60^{\circ}$ and four at $\alpha=90^{\circ}$. Symmetrical flow structures with similar rotations are observed along the diagonal of the plate at $\alpha=90^{\circ}$ (Fig. 8f).

At $\mathrm{AR}=5$, four separated tip vortices can be seen for all inclination angles (Figs. 8gi). They were not present at $\alpha=30^{\circ}$ and $\alpha=60^{\circ}$ for $A R=0.5$ and $A R=2$. This can be due to the much longer span for the $A R=5$ cases comparing to the other aspect ratios in the current study. At $\alpha=30^{\circ}$, the vortices generated from the top corners are smaller in size and are located between the vortices from the bottom corners (Fig. 8g). As inclination angle increases to $60^{\circ}$ and $90^{\circ}$, the two side tip vortices get close to each other and the two middle ones move to the top. It can be seen that at $90^{\circ}$, the tip vortices have a symmetrical pattern both in $x-y$ and $x-z$ planes.

Due to the importance of the unsteady aerodynamic loads, next section describes the variation of the unsteady loads with different aspect ratio and inclination angle configurations mentioned above.

\section{Unsteady results}

\subsection{Effect of inclination angle and aspect ratio on aerodynamic loading}

It is also of interest to investigate the behaviour of the unsteady aerodynamic loads (peak values) with respect to different aspect ratios and inclination angles. Unsteady aerodynamic loads on a flat plate play an important role in the design of structures such as solar panels and signs whose flow fields resemble that of flat plate. Due to the shape of the structure which is associated with sharp edges, unsteady aerodynamic fluctuations are inevitable. Such information can help design engineers for a better understanding of flow field and consequently manufacturing more reliable structures against wind loading.

As a measure of the unsteady aerodynamic loads, the standard deviation of the time series of drag and lift forces $\left(C_{D}\right.$, STD and $C_{L}$, STD $)$ are presented in fig. 9a, b for the aspect ratios and inclination angles evaluated in this study. These values are obtained after performing the averaging on aerodynamic loads over a large number of time steps. Figures are arranged as 3D bar charts to demonstrate the effect of changing the inclination angle while the aspect ratio is fixed as well as the effect of aspect ratio while the inclination angle is fixed. By showing the results in the form of bar chart graph, the trade-off and connection between lift and drag forces for different aspect ratios and inclination angles are demonstrated. 


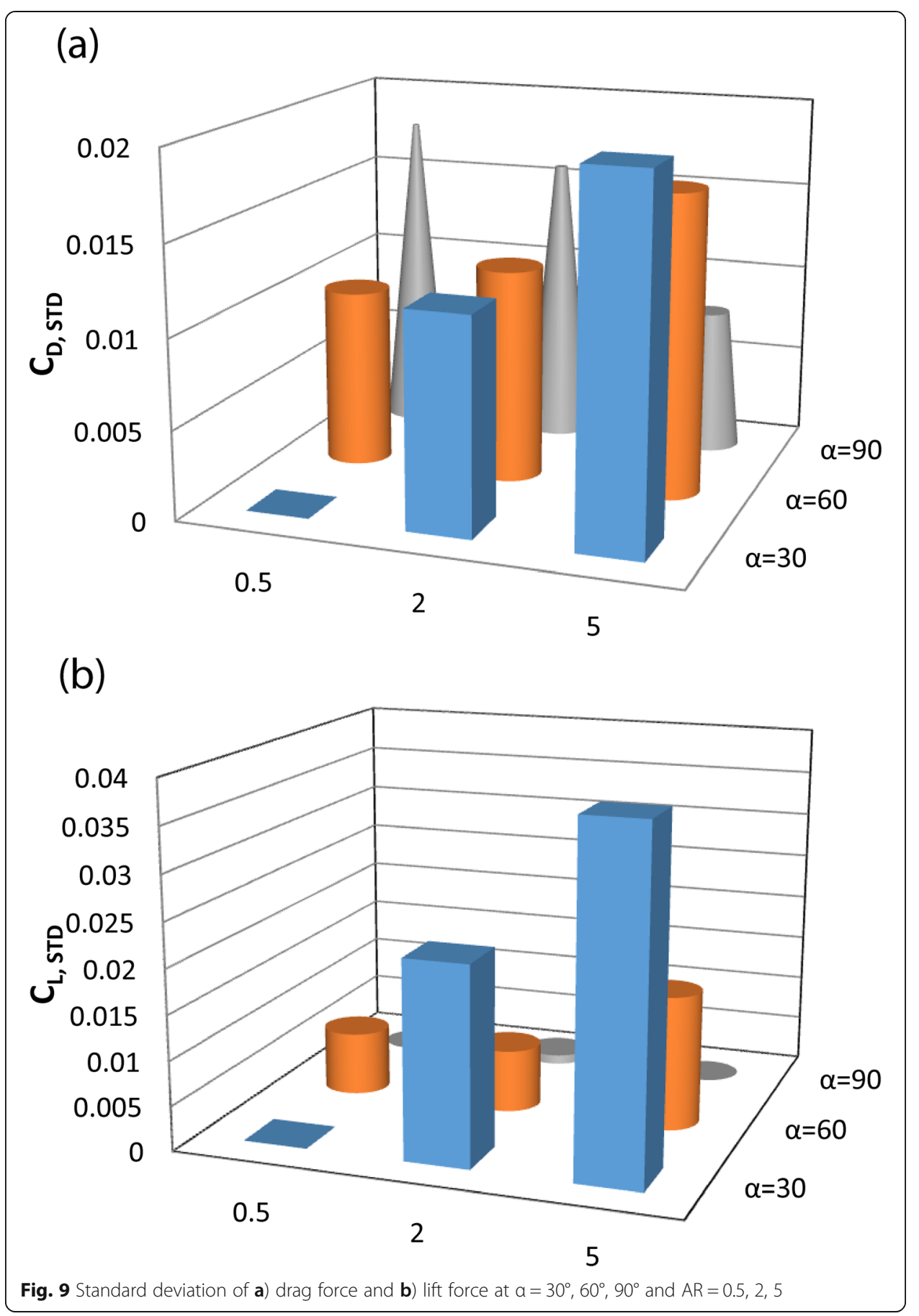

According to Fig. 9a, b, no fluctuation was observed at $\alpha=30^{\circ}$ and $A R=0.5$ case. This behavior was expected based on the presence of the two tip vortices and their influence on the control of the vortex shedding generated from the top and bottom edges. Unsteady flow visualization can explain the flow phenomenon at this specific case.

By moving to $\mathrm{AR}=2$ and $\mathrm{AR}=5$ at $\alpha=30^{\circ}$, a significant increase for both lift and drag fluctuations was seen, however, this increase is much larger for the lift coefficient $\left(0<\mathrm{C}_{\mathrm{D}}\right.$, STD $<0.02$ and $0<\mathrm{C}_{\mathrm{L}}$, STD $\left.<0.035\right)$. 
The increasing trend for the standard deviation of the aerodynamic loads continues at $\alpha=60^{\circ}$ when moving from $\mathrm{AR}=0.5$ to $\mathrm{AR}=2$ and then $\mathrm{AR}=5$.

At $\alpha=90^{\circ}$, a reducing trend for drag and no change for lift was observed when moving from $\mathrm{AR}=0.5$ to $\mathrm{AR}=5$.

Analysis of results at a fixed aspect ratio with different inclination angles reveals that, at $A R=0.5$, by increasing the inclination angle, the $C_{D}$, STD increases. Lift shows similar trend for this range except for $90^{\circ}$, which generates a value close to zero.

At $A R=2$, increasing the inclination angle shows that $C_{D}$, STD remains constant between $\alpha=30^{\circ}$ and $60^{\circ}$ and increases at $90^{\circ}$. A reducing trend can be seen for $C_{L}$, STD for this range of inclination angles.

At $A R=5$, the $C_{D}$, STD and $C_{L}$, STD are showing similar trend when increasing the inclination angle between $30^{\circ}$ and $90^{\circ}$. Both parameters are reduced when the inclination angle increases from $30^{\circ}$ to $90^{\circ}$ at this specific aspect ratio. These above-mentioned trends of variation of the unsteady aerodynamic loads with inclination and aspect ratio will be explained in Section 4.2 based on the unsteady flow structures.

Figures $9 \mathrm{a}, \mathrm{b}$ also show the configurations at which the plate experiences the maximum peak of the drag and lift forces. Therefore, these configurations can be considered as the critical cases among all the cases analyzed. It can be seen that $\alpha=30^{\circ}$ and $A R=5$ as well as the $\alpha=90^{\circ}$ and $\mathrm{AR}=0.5$ are the two cases with the highest standard deviation for the drag force. The highest peak for the lift happens at $\alpha=30^{\circ}$ and $A R=5$.

Finally, different behaviors observed for the aerodynamic fluctuations can be explained by looking at the unsteady flow structures. Next section will evaluate the connection between the standard deviation of aerodynamic loads with the flow structures in the wake region for each aspect ratio and inclination angle.

\subsection{Unsteady flow structures}

In order to explain the trend of the unsteady forces $\left(C_{D}\right.$, STD and $C_{L}$, STD $)$ observed in Figs 9a, b, unsteady flow structures behind the plate at the mentioned aspect ratios and inclination angles are visualized in Figs. 10a-i using the iso-surfaces of $\lambda_{2}$ criterion superimposed onto the streamwise vorticity contours. For each case, a large number of time steps have reached in order to better visualize the shedding of vortices in the wake region.

Figures 10a-c show the unsteady flow structures at $A R=0.5$ and $\alpha=30^{\circ}, 60^{\circ}$ and $90^{\circ}$, respectively. Comparison of the mean and unsteady $\lambda_{2}$ iso-surfaces at $\mathrm{AR}=0.5$ and $\alpha=$ $30^{\circ}$ (Figs. 8a and Figs. 10a) shows a strong similarity between the flow structures observed in these two cases, indicating a stable wake with no large scale periodicity in this case. The two clockwise and counterclockwise tip vortices prevent the propagation of the leading edge recirculation zone in the downstream direction (Fig. 8a), thus stopping the periodic shedding wake vortices for this specific case. At $\alpha=60^{\circ}$ (Fig. 10b), the effect of tip vortices is reduced and shedding starts to develop from the top and bottom edges. This separated flow from the top and bottom edges of the plate influences the tip vortices in the wake region. At $\alpha=90^{\circ}$ (Fig. 10c), due to the symmetry in geometry, the separated flow from the top and bottom edges influences similarly the tip vortices in the wake region. Therefore, the observed vortices are similar in size and rotation along the diagonal of the plate. 


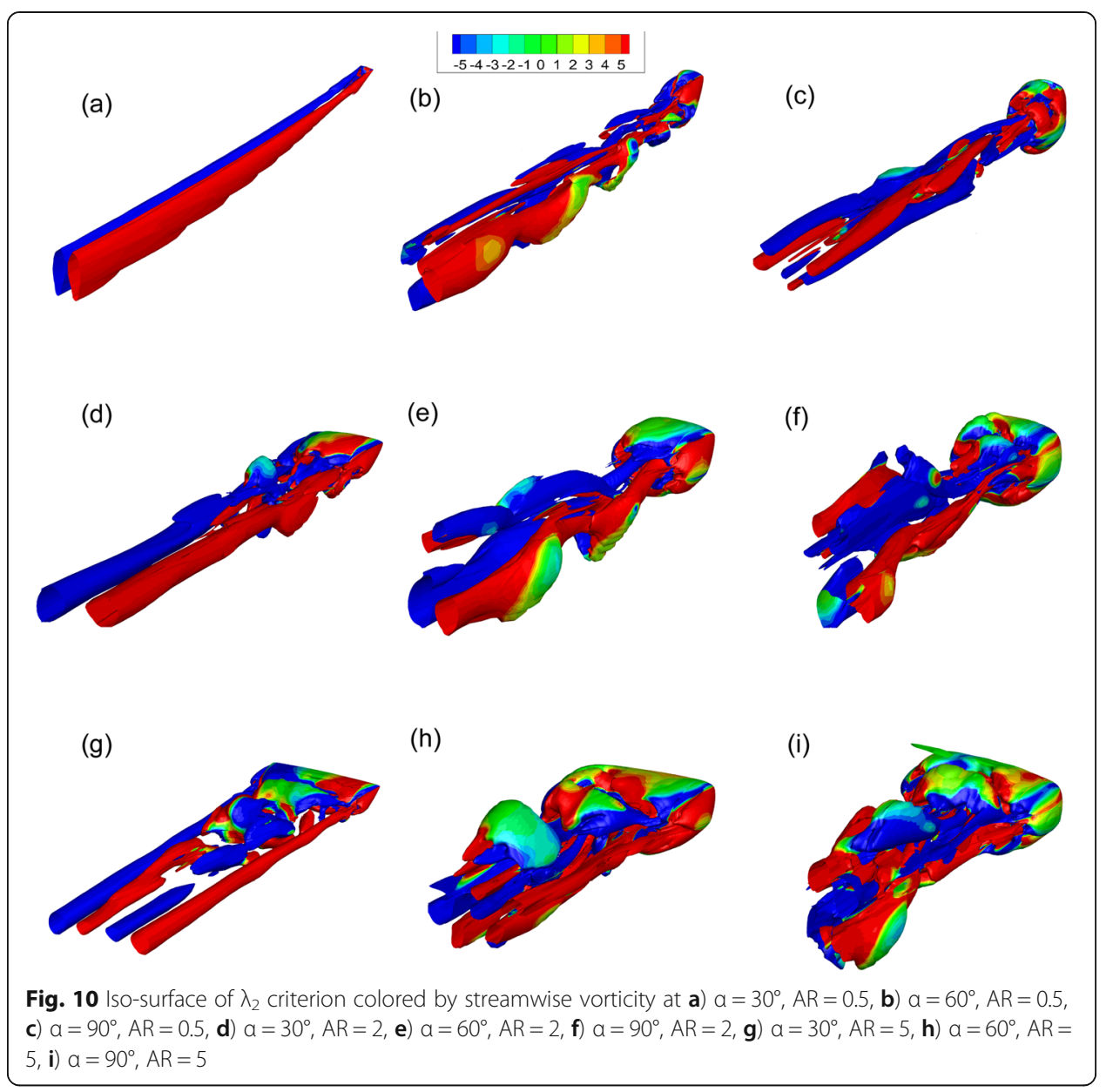

At $\mathrm{AR}=2$ and $\alpha=30^{\circ}, 60^{\circ}$, due to the larger distance between the two tip vortices compared to $\mathrm{AR}=0.5$, the leading and trailing edge vortices have more freedom for interaction and therefore periodic shedding results in a vortex street. The tip vortices are more influenced by the separating flow from the top and bottom edges at $\alpha=60^{\circ}$ than $\alpha=30^{\circ}$ due to the stronger interaction at this inclination angle. At $\alpha=90^{\circ}$, identical separated flow from the top and bottom edges performs similarly in influencing the vortices in the wake region. The tip vortices are significantly influenced and have almost lost their round shape comparing to other cases $\left(\alpha=30^{\circ}, 60^{\circ}\right)$.

At $A R=5$ (Figs. 10g-i), the influence of the tip vortices on the leading and trailing edge vortices due to larger span relative to other aspect ratios is further reduced, and the leading and trailing edge vortices shed into the wake without significant decay or cancellation due to the effect of the tip vortices. As a result, the body is exposed to higher fluctuations of the unsteady aerodynamic loads, as evidenced by the standard deviation values in Figs 9a, b. At $\alpha=30^{\circ}$, the separated flow from the top and bottom edges has insignificant influence on the tip vortices. As observed in Fig. 10g, the tip vortices keep their round shape at this specific inclination angle. At $\alpha=60^{\circ}$, since the tip vortices get close to each other, they are influenced by separated flow generated from top and bottom edges. This interaction is more severe at $\alpha=90^{\circ}$, where the tip 
vortices are significantly influenced by separated flow. As a result, these vortices have lost their round shape at this specific aspect ratio and inclination angle.

In general, the results show a correlation between the intensity of the unsteady aerodynamic loads, and the influence of the tip vortices on the leading edge and trailing edge vortices, which is decreased in the cases with higher aspect ratios, where the spacing between the tip vortices is larger. Basically, the larger the distance between the tip vortices is, the stronger happens the shedding of leading and trailing edges and unsteady loads.

As illustrated in the presented figures, the distance between tip vortices is a key factor for controlling the strength of the shedding vortices and unsteady aerodynamic fluctuations.

\section{Conclusion}

Large Eddy Simulations have been carried out to investigate flow structures and unsteady aerodynamic loads of a thin flat plate at different geometric and flow configurations represented by different aspect ratios and inclination. For validation purposes, results obtained from the numerical model have been compared with the available experimental data. The good agreement observed between the results confirms the validity of the numerical model.

Analysis of the mean flow results shows a strong dependence of the aerodynamic forces with the aspect ratio and inclination angle. Increase of the inclination angle at each aspect ratio increases drag and reduces the lift force. Increasing the aspect ratio while the inclination angle is constant shows an increase in drag while the lift force remains unchanged.

Among all the aspect ratios and inclination angles investigated, the $\mathrm{AR}=0.5$ case at $\alpha=30^{\circ}$ shows an irregular behavior where the drag and lift are found to be maximum in that specific combination of inclination angle and aspect ratio.

The $\lambda_{2}$ iso-surfaces superimposed with streamwise vorticity contours were used to visualize the mean and unsteady flow structures. The iso-surfaces of $\lambda_{2}$ criterion show how the inclination angle and aspect ratio parameters influence the vortex shedding phenomenon.

A stable wake with the presence of two tip vortices is observed at $\mathrm{AR}=0.5, \alpha=30^{\circ}$ which results in minimal fluctuation of aerodynamic forces at this specific case. At $\alpha=$ $60^{\circ}$, also two tip vortices were present and at $\alpha=90^{\circ}$, due to the symmetry, four vortices appeared. At $A R=2$, due to the longer span, the leading and trailing edge vortices have more freedom for shedding and consequently larger unsteady aerodynamic forces are observed for those cases. At $A R=5$, the span is even longer than the other aspect ratios and this results in more freedom for flow moving from leading and trailing edge to influence the wake. The four tip vortices which are present in all inclination angles at this aspect ratio interact with the flow from leading and trailing edge, and as a result, large aerodynamic force fluctuations were observed comparing to other aspect ratios.

In general, the results show a correlation between the intensity of the unsteady aerodynamic loads, and the influence of the tip vortices on the leading edge and trailing edge vortices, which is decreased in the cases with higher aspect ratios, where the spacing between the tip vortices is larger. Basically, for cases with a larger gap between the tip vortices, the leading and trailing edge vortices have more freedom for shedding and 
consequently larger unsteady aerodynamic forces are observed for those cases, while for cases with smaller gap, the shedding from leading and trailing edges are weakened and controlled.

\section{Acknowledgements}

The first author would like to acknowledge the support of the Natural Sciences and Engineering Research Council of Canada (NSERC) through the Postdoctoral Fellowship (PDF) program.

\section{Authors' contributions}

Both authors have performed equally during the manuscript preparation. The authors read and approved the final manuscript.

\section{Funding}

This study was supported by the Natural Sciences and Engineering Research Council of Canada (NSERC) through the Postdoctoral Fellowship (PDF) program.

\section{Availability of data and materials}

The files supporting the results of this article are available upon request.

\section{Competing interests}

Authors declare that they have no conflict of interest.

Received: 24 February 2020 Accepted: 30 April 2020

Published online: 19 June 2020

\section{References}

1. Fage A, Johansen FC (1927) On the flow of air behind an inclined flat plate of infinite span. Proc R Soc Lond 116:170197

2. Flachsbart O (1932) Messungen an ebenen und gewölbten Platten. Ergebnisse der Aerodynamischen Versuchsanstalt zu Göttingen 4:96-100

3. Schubauer GB, Dryden HL (1937) The effect of turbulence on the drag of flat plates. NACA Report 546

4. Roshko A (1954) On the drag and shedding frequency of two-dimensional bluff bodies. NACA Tech Note 3169

5. Arie $M$, Rouse $H$ (1956) Experiments on two-dimensional flow over a normal wall. J Fluid Mech 1:129-141

6. Fail R, Lawford JA, Eyre RCW (1959) Low speed experiments on the wake characteristics of flat plates normal to an air stream. Aeronautical Research Council Reports and Memoranda

7. Abernathy FH (1962) Flow over an inclined plate. ASME J Basic Eng 61:124

8. Castro IP (1971) Wake characteristics of two-dimensional perforated plates normal to an air-stream. J Fluid Mech 46: 599-609

9. Sarpkaya T, Kline HK (1982) Impulsively-started flow about four types of bluff body. J Fluids Eng 104:207-213

10. Kiya M, Matsumura M (1988) Incoherent turbulence structure in the near wake of a normal plate. J Fluid Mech 190:343356

11. Mazharoglu C, Hacisevki H (1999) Coherent and incoherent flow structures behind a normal flat plate. Exp Thermal Fluid Sci 19(3):160-167

12. Xu Y, Feng L, Wang J (2015) Experimental investigation on the flow over normal flat plates with various corner shapes. J Turbul 16(7):607-616

13. Tamada K, Miyagi T (1962) Laminar viscous flow past a flat plate set normal to the stream, with special reference to high Reynolds numbers. J Phys Soc Jpn 17:373-390

14. Kuwahara K (1973) Numerical study of flow past an inclined flat plate by an inviscid model. J Phys Soc Jpn 35:5

15. Sarpkaya T (1975) An inviscid model of two diemensional vortex shedding for transient and asymptotically steady separated flow over an inclined plate. J Fluid Mech 68:109-128

16. Hudson JD, Dennis SCR (1985) The flow of a viscous incompressible fluid past a normal flat plate at low and intermediate Reynolds numbers: the wake. J Fluid Mech 160:369

17. Chein R, Chung JN (1988) Discrete vortex simulation of flow over inclined and normal plates. Comput Fluids 16(4):405427

18. Chua K, Lisoski D, Leonard A, Roshko A (1990) A numerical and experimental investigation of separated flow past an oscillating flat plate. In: ASME FED international symposium on non-steady fluid dynamics, book no. H00597 92, pp 455464

19. Dennis SCR, Qiang W, Coutanceau M, Launay JL (1993) Viscous flow normal to a flat plate at moderate Reynolds numbers. J Fluid Mech 248:605-635

20. Tamaddon-Jahromi HR, Townsend P, Webster MF (1994) Unsteady viscous flow past a flat plate orthogonal to the flow. Comput Fluids 23:433-446

21. Najjar FM, Vanka SP (1995) Simulations of the unsteady separated flow past a normal flat plate. Int J Numer Methods Fluids 21(7):525-547

22. Najjar FM, Vanka SP (1995) Effects of intrinsic three-dimensionality on the drag characteristics of a normal flat plate. Phys Fluids 7:2516-2518

23. Yeung WWH, Parkinson GV (1997) On the steady separated flow around an inclined flat plate. J Fluid Mech 333:403-413

24. Najjar FM, Balachandar S (1998) Low-frequency unsteadiness in the wake of a normal flat plate. J Fluid Mech 370:101

25. Lasher WC (2001) Computation of two-dimensional blocked flow normal to a flat plate. J Wind Eng Ind Aerodyn 89: 493-513 
26. Narasimhamurthy VD, Andersson HI (2009) Numerical simulation of the turbulent wake behind a normal flat plate. Int J Heat Fluid Flow 30:1037-1043

27. Taira K, Colonius T (2009) Three-dimensional flows around low-aspect-ratio flat-plate wings at low Reynolds numbers. J Fluid Mech 623:187-207

28. Afgan I, Benhamadouche S, Han X, Sagaut P, Laurence D (2013) Flow over a flat plate with uniform inlet and incident coherent gusts. J Fluid Mech 720:457-485

29. Yang D, Pettersen B, Andersson HI, Narasimhamurthy VD (2013) On oblique and parallel shedding behind an inclined plate. Phys Fluids 25:054101

30. Tian X, Ong MC, Yang J, Myrhaug D (2014) Large-eddy simulation of the flow normal to a flat plate including corner effects at a high Reynolds number. J Fluids Struct 49:149-169

31. Hemmati A, Wood DH, Martinuzzi RJ (2016) Characteristics of distinct flow regimes in the wake of an infinite span normal thin flat plate. Int J Heat Fluid Flow 62:423-436

32. Chen YS, Kim SW (1987) Computation of turbulent flows using an extended k-epsilon turbulence closure model. Technical Report NASA-CR-179204

33. Yakhot Y, Orszag SA, Thangam S, Gatski TB, Speziale CG (1992) Development of turbulence models for shear flows by a double expansion technique. Phys Fluids A 4(7):1510-1520

34. Speziale CG (1987) On nonlinear K-I and K- $\varepsilon$ models of turbulence. J Fluid Mech 178:459-475

35. Takeuchi M, Okamoto T (1983) Effect of side walls of wind-tunnel on turbulent wake behind two-dimensional bluff body. In: Bradbury LJS (ed) Proceedings of the 4th symposium on turbulent shear flows. Springer-Verlag, Karlsruhe, pp 525-530

36. Shademan M, Hangan H (2009) Wind loading on solar panels at different inclination angles. In: 11th conference of American Society of Wind Engineers, Puerto Rico

37. Shademan M, Hangan H (2010) Wind loading on solar panels at different azimuthal and inclination angles. In: Fifth international symposium on computational wind engineering (CWE2010), North Carolina, USA

38. Shademan M, Barron RM, Balachandar R, Hangan H (2014) Numerical simulation of wind loading on ground-mounted solar panels at different flow configuration. Can J Civ Eng 41(8):728-738

39. Shademan M, Balachandar R, Barron RM (2014) Detached eddy simulation of flow past an isolated inclined solar panel. $J$ Fluids Struct 50:217-230

40. Bosch G, Rodi W (1996) Simulation of vortex shedding past a square cylinder near a wall. Int J Heat Fluid Flow 17(3): $267-275$

41. Franke J, Hellsten A, Schlunzen H, Carissimo B (2007) Best practice guideline for the CFD simulation of flows in the urban environment: COST action 732, quality assurance and improvement of micro-scale meteorological models

42. Piomelli U, Chasnov JR (1996) Large-eddy simulations: theory and applications. In: Henningson A, Hallback K, Alfredsson $\mathrm{L}$, Johansson M (eds) Transition and turbulence modelling. Kluwer Academic Publishers, Dordrecht, p 269

43. Germano M, Piomelli U, Moin P, Cabot WH (1991) A dynamic subgrid-scale eddy viscosity model. Phys Fluids A3:17601765

44. OpenCFD Limited, http://www.openfoam.org

45. Jeong J, Hussain F (1995) On the identification of a vortex. J Fluid Mech 285:69-94

\section{Publisher's Note}

Springer Nature remains neutral with regard to jurisdictional claims in published maps and institutional affiliations.

\section{Submit your manuscript to a SpringerOpen ${ }^{\circ}$ journal and benefit from:}

- Convenient online submission

- Rigorous peer review

- Open access: articles freely available online

- High visibility within the field

- Retaining the copyright to your article

Submit your next manuscript at $>$ springeropen.com 